\title{
New results on positive almost periodic solutions for first-order neutral differential equations
}

\author{
Yuehua Yu' and Shuhua Gong ${ }^{2^{*}}$ (1)
}

\section{"Correspondence:}

shuhuagong@aliyun.com

${ }^{2}$ College of Mathematics, Physics

and Information Engineering,

Jiaxing University, Jiaxing, P.R. China

Full list of author information is

available at the end of the article

\begin{abstract}
In this paper, a class of first-order neutral differential equations with time-varying delays and coefficients is considered. Some results on the existence of positive almost periodic solutions for the equations are obtained by using the contracting mapping principle and the differential inequality technique. In addition, an example is given to illustrate our results.
\end{abstract}

MSC: $34 \mathrm{C} 25 ; 34 \mathrm{~K} 13$

Keywords: Positive almost periodic solution; First order; Neutral differential equation

\section{Introduction}

In recent years, the following first-order neutral differential equations

$$
[x(t)-P(t) x(t-r)]^{\prime}=-Q(t) x(t)+f(t, x(t-r))
$$

and

$$
[x(t)-c x(t-\tau(t))]^{\prime}=-Q(t) x(t)+f(t, x(t-\tau(t)))
$$

have been extensively used to describe the dynamic behaviors for the blood cell production models, population models, and control models (see, for example, [1-5] and the references therein). Here, $Q, \tau \in C(\mathbb{R},(0,+\infty)), P \in C^{1}(\mathbb{R}, \mathbb{R}), f \in C(\mathbb{R} \times \mathbb{R}, \mathbb{R}), r>0$, and $|c|<1$ are constants. In particular, assuming that $P, Q$ are $\omega$-periodic functions, $f$ is $\omega$-periodic with respect to the first variable, and

$$
\inf _{t \in \mathbb{R}} Q(t)>0
$$

some criteria ensuring the existence on the positive periodic solutions of (1.1) and (1.2) have been established in [6] and [7], respectively. It is well known that almost periodically variable coefficients and delays in differential equations of population and ecology problems are much more realistic in the real world. Therefore, in recent years, there has been increasing interest in the existence and stability of almost periodic type solutions for firstorder functional differential equations in population models [8-12]. However, to the best

(c) The Author(s) 2018. This article is distributed under the terms of the Creative Commons Attribution 4.0 International License (http://creativecommons.org/licenses/by/4.0/), which permits unrestricted use, distribution, and reproduction in any medium, provided you give appropriate credit to the original author(s) and the source, provide a link to the Creative Commons license, and indicate if changes were made. 
of our knowledge, there are few papers published on positive almost periodic solutions of (1.1) and (1.2). Motivated by the above discussions, in this paper we aim to establish some sufficient conditions on the existence of positive almost periodic solutions of the following first-order neutral differential equations with time-varying delays and coefficients:

$$
\left[x(t)-P(t) x\left(t-\tau_{1}(t)\right)\right]^{\prime}=-Q(t) x(t)+f\left(t, x\left(t-\tau_{2}(t)\right)\right),
$$

where $Q, P \in C(\mathbb{R},(0,+\infty)), \tau_{1}, \tau_{2} \in C(\mathbb{R},[0,+\infty))$ are almost periodic functions, $f \in C(\mathbb{R} \times$ $\mathbb{R}, \mathbb{R})$ is an almost periodic function for $t$ uniformly on $\mathbb{R}$, and

$$
M[Q]=\lim _{T \rightarrow+\infty} \frac{1}{T} \int_{t}^{t+T} Q(s) d s>0,
$$

where the limit above is independent of $t$. The contributions of this paper can be summarized as follows: (1) In this manuscript, all delays and coefficients of (1.4) are time-varying, and (1.1) and (1.2) are special cases of (1.4); (2) The sufficient conditions for the existence of positive almost periodic solution are derived in terms of its coefficients without (1.3), which has not been investigated till now.

Throughout this paper, we denote the set of almost periodic functions from $\mathbb{R}$ to $\mathbb{R}$ by $A P(\mathbb{R}, \mathbb{R})$. Then, $\left(A P(\mathbb{R}, \mathbb{R}),\|\cdot\|_{\infty}\right)$ is a Banach space, where $\|\cdot\|_{\infty}$ denotes the supremum $\|u\|_{\infty}:=\sup _{t \in \mathbb{R}}|u(t)|$. For more details, we refer the reader to $[13,14]$.

\section{Main results}

Theorem 2.1 Let $\tau_{1}(t) \not \equiv \tau_{2}(t)$ for all $t \in \mathbb{R}$, and assume that the following conditions hold:

$\left(A_{1}\right)$ There exist positive constants $F^{S}, F^{i}$ and a bounded and continuous function $Q^{*}$ : $\mathbb{R} \rightarrow(0,+\infty)$ such that

$$
F^{i} e^{-\int_{s}^{t} Q^{*}(u) d u} \leq e^{-\int_{s}^{t} Q(u) d u} \leq F^{S} e^{-\int_{s}^{t} Q^{*}(u) d u} \quad \text { for all } t, s \in \mathbb{R} \text { and } t-s \geq 0,
$$

where $Q^{*}$ has the lower bound different from zero.

$\left(A_{2}\right)$ There exist positive constants $p_{0}, p_{1}, m$, and $M$ such that

$$
\left\{\begin{array}{l}
0 \leq p_{0}=\inf _{t \in \mathbb{R}} P(t) \leq \sup _{t \in \mathbb{R}} P(t)=p_{1}, \\
\sup _{t \in \mathbb{R}, x, y \in[m, M]} \frac{F^{S}[-Q(t) P(t) x+f(t, y)]}{Q^{*}(t)} \leq\left(1-p_{1}\right) M \\
\inf _{t \in \mathbb{R}, x, y \in[m, M]} \frac{F^{i}[-Q(t) P(t) x+f(t, y)]}{Q^{*}(t)} \geq\left(1-p_{0}\right) m .
\end{array}\right.
$$

$\left(A_{3}\right)$ There exist positive constants $L^{f}$ and $L$ such that $L+p_{1}<1$,

$$
\begin{aligned}
& \sup _{t \in \mathbb{R}} F^{S} \frac{|Q(t) P(t)|+L^{f}}{Q^{*}(t)} \leq L \text { and } \\
& \left|f\left(t, x_{1}\right)-f\left(t, x_{2}\right)\right| \leq L^{f}\left|x_{1}-x_{2}\right| \text { for all } t, x_{1}, x_{2} \in \mathbb{R} .
\end{aligned}
$$

Then equation (1.4) has at least one positive almost periodic solution $x^{*}$ such that $x^{*}(t) \in[m, M]$ for all $t \in \mathbb{R}$.

Proof Set

$$
B=\{\varphi \mid \varphi \in A P(\mathbb{R}, \mathbb{R}), m \leq \varphi(t) \leq M \text { for all } t \in \mathbb{R}\} .
$$


Clearly, $B$ is a closed subset of $A P(\mathbb{R}, \mathbb{R})$. For any $\varphi \in B$, we consider an auxiliary equation

$$
x^{\prime}(t)=-Q(t) x(t)-Q(t) P(t) \varphi\left(t-\tau_{1}(t)\right)+f\left(t, \varphi\left(t-\tau_{2}(t)\right)\right) .
$$

In view of the fact that $M[Q]>0$, it follows from Theorem 7.7 of [13] that system (2.4) has exactly one almost periodic solution

$$
x^{\varphi}(t)=\int_{-\infty}^{t} e^{-\int_{s}^{t} Q(u) d u}\left[-Q(s) P(s) \varphi\left(s-\tau_{1}(s)\right)+f\left(s, \varphi\left(s-\tau_{2}(s)\right)\right)\right] d s, \quad \forall \varphi \in B,
$$

where

$$
\begin{aligned}
{\left[x^{\varphi}(t)\right]^{\prime}=} & \left\{\int_{-\infty}^{t} e^{-\int_{s}^{t} Q(u) d u}\left[-Q(s) P(s) \varphi\left(s-\tau_{1}(s)\right)+f\left(s, \varphi\left(s-\tau_{2}(s)\right)\right)\right] d s\right\}^{\prime} \\
= & -Q(t)\left\{\int_{-\infty}^{t} e^{-\int_{s}^{t} Q(u) d u}\left[-Q(s) P(s) \varphi\left(s-\tau_{1}(s)\right)+f\left(s, \varphi\left(s-\tau_{2}(s)\right)\right)\right] d s\right\} \\
& -Q(t) P(t) \varphi\left(t-\tau_{1}(t)\right)+f\left(t, \varphi\left(t-\tau_{2}(t)\right)\right) \\
= & -Q(t) x^{\varphi}(t)-Q(t) P(t) \varphi\left(t-\tau_{1}(t)\right)+f\left(t, \varphi\left(t-\tau_{2}(t)\right)\right) .
\end{aligned}
$$

In view of $P, \tau_{1} \in A P(\mathbb{R}, \mathbb{R})$ and Lemma 2.4 in [15], we obtain

$$
P(t) \varphi\left(t-\tau_{1}(t)\right) \in A P(\mathbb{R}, \mathbb{R}), \quad \forall \varphi \in B .
$$

Now, we define a mapping $T: B \rightarrow A P(\mathbb{R}, \mathbb{R})$ as follows:

$$
(T \varphi)(t)=P(t) \varphi\left(t-\tau_{1}(t)\right)+x^{\varphi}(t), \quad \forall \varphi \in B .
$$

Next, we will prove that the mapping $T$ is a contraction mapping on $B$.

For all $t \in \mathbb{R}$, according to $\left(A_{1}\right)$ and $\left(A_{2}\right)$, we have

$$
\begin{aligned}
& P(t) \varphi\left(t-\tau_{1}(t)\right)+x^{\varphi}(t) \\
& \leq p_{1} M+\int_{-\infty}^{t} e^{-\int_{s}^{t} Q^{*}(u) d u} F^{S}\left[-Q(s) P(s) \varphi\left(s-\tau_{1}(s)\right)\right. \\
& \quad\left.+f\left(s, \varphi\left(s-\tau_{2}(s)\right)\right)\right] d s \\
& \leq p_{1} M+\int_{-\infty}^{t} e^{-\int_{s}^{t} Q^{*}(u) d u}\left(1-p_{1}\right) M Q^{*}(s) d s \leq M
\end{aligned}
$$

and

$$
\begin{aligned}
& P(t) \varphi\left(t-\tau_{1}(t)\right)+x^{\varphi}(t) \\
& \geq p_{0} m+\int_{-\infty}^{t} e^{-\int_{s}^{t} Q^{*}(u) d u} F^{i}\left[-Q(s) P(s) \varphi\left(s-\tau_{1}(s)\right)\right. \\
& \left.\quad+f\left(s, \varphi\left(s-\tau_{2}(s)\right)\right)\right] d s \\
& \geq p_{0} m+\int_{-\infty}^{t} e^{-\int_{s}^{t} Q^{*}(u) d u}\left(1-p_{0}\right) m Q^{*}(s) d s \geq m,
\end{aligned}
$$

which imply that the mapping $T$ is a self-mapping from $B$ to $B$. 
Furthermore, for all $\varphi, \psi \in B,(2.5),\left(A_{1}\right)$ and $\left(A_{3}\right)$ yield

$$
\begin{aligned}
\| T \varphi & -T \psi \|_{\infty} \\
\leq & \sup _{t \in \mathbb{R}}\left\{\left|P(t)\left[\varphi\left(t-\tau_{1}(t)\right)-\psi\left(t-\tau_{1}(t)\right)\right]\right|\right. \\
& +\int_{-\infty}^{t} e^{-\int_{s}^{t} Q^{*}(u) d u} F^{S} \mid-Q(s) P(s)\left(\varphi\left(s-\tau_{1}(s)\right)-\psi\left(s-\tau_{1}(s)\right)\right) \\
& \left.+\left(f\left(s, \varphi\left(s-\tau_{2}(s)\right)\right)-f\left(s, \psi\left(s-\tau_{2}(s)\right)\right)\right) \mid d s\right\} \\
\leq & \|\varphi-\psi\|_{\infty}\left\{p_{1}+\sup _{t \in \mathbb{R}} \int_{-\infty}^{t} e^{-\int_{s}^{t} Q^{*}(u) d u} F^{S}\left[|Q(s) P(s)|+L^{f}\right] d s\right\} \\
\leq & \|\varphi-\psi\|_{\infty}\left[p_{1}+\sup _{t \in \mathbb{R}} \int_{-\infty}^{t} e^{-\int_{s}^{t} Q^{*}(u) d u} Q^{*}(s) L d s\right] \\
\leq & \left(p_{1}+L\right)\|\varphi-\psi\|_{\infty} .
\end{aligned}
$$

Thus, the mapping $T$ is a contraction on $B$. Using the classical contraction mapping principle of Banach-Caccioppoli, we obtain that the mapping $T$ possesses a unique fixed point $x^{*} \in B, T x^{*}=x^{*}$, i.e.,

$$
\begin{aligned}
x^{*}(t)= & P(t) x^{*}\left(t-\tau_{1}(t)\right)+x^{x^{*}}(t) \\
= & P(t) x^{*}\left(t-\tau_{1}(t)\right) \\
& +\int_{-\infty}^{t} e^{-\int_{s}^{t} Q(u) d u}\left[-Q(s) P(s) x^{*}\left(s-\tau_{1}(s)\right)+f\left(s, x^{*}\left(s-\tau_{2}(s)\right)\right)\right] d s,
\end{aligned}
$$

which together with (2.6) leads to

$$
\left[x^{*}(t)-P(t) x^{*}\left(t-\tau_{1}(t)\right)\right]^{\prime}=-Q(t) x^{*}(t)+f\left(t, x^{*}\left(t-\tau_{2}(t)\right)\right) .
$$

This completes the proof.

Remark 2.1 When $\tau_{1}(t) \equiv \tau_{2}(t)$ for all $t \in \mathbb{R}$, the statement of Theorem 2.1 remains valid if we replace $\left(A_{2}\right)$ by the following condition:

$\left(A_{2}^{*}\right)$ There exist positive constants $p_{0}, p_{1}, m$, and $M$ such that

$$
\left\{\begin{array}{l}
0 \leq p_{0}=\inf _{t \in \mathbb{R}} P(t) \leq \sup _{t \in \mathbb{R}} P(t)=p_{1} \\
\sup _{t \in \mathbb{R}, x \in[m, M]} \frac{F^{S}[-Q(t) P(t) x+f(t, x)]}{Q^{*}(t)} \leq\left(1-p_{1}\right) M \\
\inf _{t \in \mathbb{R}, x \in[m, M]} \frac{F^{i}[-Q(t) P(t) x+f(t, x)]}{Q^{*}(t)} \geq\left(1-p_{0}\right) m
\end{array}\right.
$$

Theorem 2.2 Suppose $\left(A_{1}\right)$ and $\left(A_{3}\right)$ hold. If $\tau_{1}(t) \not \equiv \tau_{2}(t)$ for all $t \in \mathbb{R}$, and the following condition holds:

$\left(\bar{A}_{2}\right)$ There exist positive constants $p_{0}, p_{1}, m$, and $M$ such that

$$
\left\{\begin{array}{l}
-p_{1}=\inf _{t \in \mathbb{R}} P(t) \leq \sup _{t \in \mathbb{R}} P(t)=-p_{0} \leq 0, \\
\sup _{t \in \mathbb{R}, x, y \in[m, M]} \frac{F^{S}[-Q(t) P(t) x+f(t, y)]}{Q^{*}(t)} \leq M+p_{0} m, \\
\inf _{t \in \mathbb{R}, x, y \in[m, M]} \frac{\left.F^{i}[Q(t))(t) x+f(t, y)\right]}{Q^{*}(t)} \geq m+p_{1} M .
\end{array}\right.
$$


Then equation (1.4) has at least one positive almost periodic solution $x^{*}$ such that $x^{*}(t) \in[m, M]$ for all $t \in \mathbb{R}$.

Remark 2.2 When $\tau_{1}(t) \equiv \tau_{2}(t)$ for all $t \in \mathbb{R}$, the statement of Theorem 2.2 holds if we substitute $\left(\bar{A}_{2}\right)$ into the following condition:

$\left(\bar{A}_{2}^{*}\right)$ There exist positive constants $p_{0}, p_{1}, m$, and $M$ such that

$$
\left\{\begin{array}{l}
-p_{1}=\inf _{t \in \mathbb{R}} P(t) \leq \sup _{t \in \mathbb{R}} P(t)=-p_{0} \leq 0 \\
\sup _{t \in \mathbb{R}, x \in[m, M]} \frac{F^{S}[-Q(t) P(t) x+f(t, x)]}{Q^{*}(t)} \leq M+p_{0} m \\
\inf _{t \in \mathbb{R}, x \in[m, M]} \frac{F^{i}[-Q(t) P(t) x+f(t, x)]}{Q^{*}(t)} \geq m+p_{1} M
\end{array}\right.
$$

\section{An example}

Example 3.1 Consider the following first-order neutral differential equations with timevarying delays and coefficients:

$$
\begin{aligned}
& {\left[x(t)-\frac{\sin ^{2} t}{100} x\left(t-\left(1+\sin ^{2} t\right)\right)\right]^{\prime}} \\
& \quad=-(1+2 \sin 400 t) x(t)+20+e^{\cos \sqrt{2} t}+\frac{1}{100} \cos x\left(t-\left(1+\sin ^{2} \sqrt{3} t\right)\right),
\end{aligned}
$$

where

$$
\left\{\begin{array}{l}
P(t)=\frac{\sin ^{2} t}{100}, \quad p_{0}=0, \quad p_{1}=\frac{1}{100}, \\
\tau_{1}(t)=1+\sin ^{2} t, \quad \tau_{2}(t)=1+\sin ^{2} \sqrt{3} t, \\
Q(t)=1+2 \sin 400 t, \quad Q^{*}(t)=1, \quad M[Q]=1, \\
F^{S}=e^{\frac{1}{100}}, \quad F^{i}=e^{-\frac{1}{100},} \\
f(t, x)=20+e^{\cos \sqrt{2} t}+\frac{1}{100} \cos x, \quad L^{f}=\frac{1}{100}, \quad L=\frac{1}{25} .
\end{array}\right.
$$

Taking $m=10, M=40$, we can easily show that (3.2) implies that (3.1) satisfies $\left(A_{1}\right),\left(A_{2}\right)$, and $\left(A_{3}\right)$. Hence, equation (3.1) has exactly one positive almost periodic solution $x^{*}(t)$.

Remark 3.1 In equation (3.1), $\tau_{1}(t)=1+\sin ^{2} t$ and $\tau_{2}(t)=1+\sin ^{2} \sqrt{3} t$ are two different time-varying functions, and $Q(t)=1+2 \sin 400 t$ fails to satisfy (1.3). One can see that all the results obtained in $[1-12,15]$ are invalid for $(3.1)$. Note that the space of almost periodic functions contains the space of periodic functions. If we reduce all time-varying delays and coefficients of (1.4) to $\omega$-periodic functions, the derived results are still novel.

Figure 1 Numerical solutions $x(t)$ of systems (3.1) for initial values $x(0)=11,23,32$, respectively

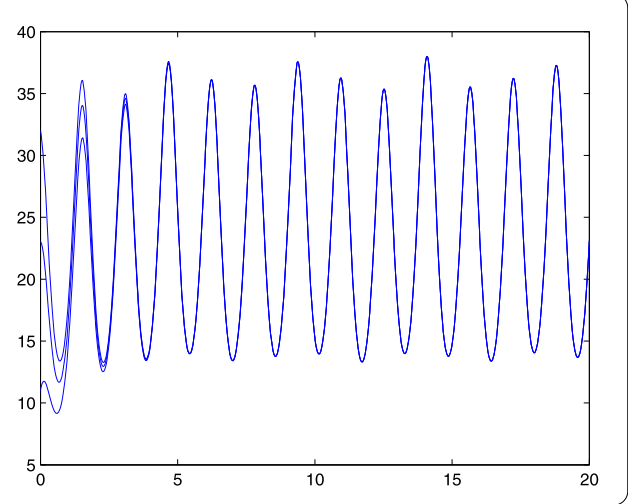




\section{Conclusion}

It is well known that the existence of positive almost periodic solutions plays an important role in characterizing the behavior of nonlinear differential equations. Thus it has been extensively investigated by numerous scholars in recent years. In this article, we have investigated a class of first-order neutral differential equations with time-varying delays and coefficients. With the aid of the contraction mapping fixed point theorem and differential inequality theory, some sufficient conditions for the existence of positive almost periodic solutions of the system were established. In order to demonstrate the usefulness of the presented results, an illustrative example was given. The established results were compared with those of recent methods existing in the literature. We expect to extend this work to more types of neutral differential equations with almost periodic delays and coefficients.

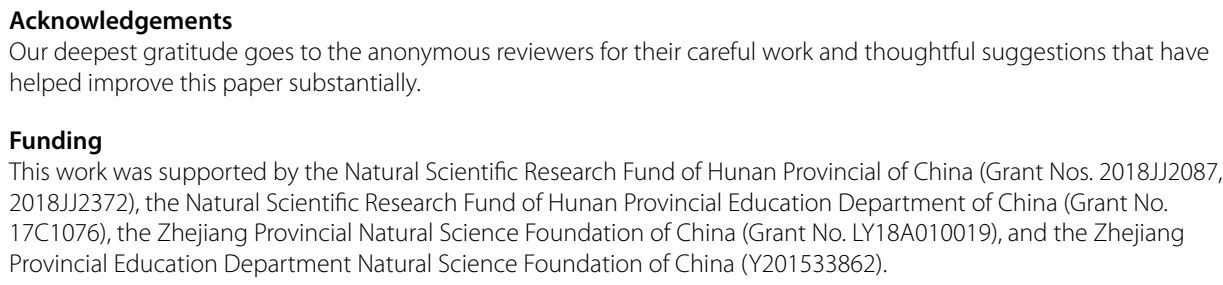

\section{Author details}

${ }^{1}$ College of Mathematics and Computer Science, Hunan University of Arts and Science, Changde, P.R. China. ${ }^{2}$ College of Mathematics, Physics and Information Engineering, Jiaxing University, Jiaxing, P.R. China.

\section{Publisher's Note}

Springer Nature remains neutral with regard to jurisdictional claims in published maps and institutional affiliations.

Received: 1 January 2018 Accepted: 7 May 2018 Published online: 21 May 2018

\section{References}

1. Olach, R.: Positive periodic solutions of delay differential equations. Appl. Math. Lett. 26, 1141-1145 (2013)

2. Graef, J.R., Kong, L.: Periodic solutions of first order functional differential equations. Appl. Math. Lett. 24, 1981-1985 (2011)

3. Liu, B., Huang, L.: Existence and uniqueness of periodic solutions for a kind of first order neutral functional differential equations. J. Math. Anal. Appl. 322(1), 121-132 (2006)

4. Liu, B., Huang, L.: Existence and uniqueness of periodic solutions for a kind of first order neutral functional differential equations with a deviating argument. Taiwan. J. Math. 11(2), 497-510 (2007)

5. Peng, L., Wang, L.: Periodic solutions for first order neutral functional differential equations with multiple deviating arguments. Ann. Pol. Math. 111(2), 197-213 (2014)

6. Luo, Y., Wang, W., Shen, J.: Existence of positive periodic solutions for two kinds of neutral functional differential equations. Appl. Math. Lett. 21, 581-587 (2008)

7. Candan, T.: Existence of positive periodic solutions of first order neutral differential equations with variable coefficients. Appl. Math. Lett. 52, 142-148 (2016)

8. Zhang, H., Yang, M., Wang, L.: Existence and exponential convergence of the positive almost periodic solution for a model of hematopoiesis. Appl. Math. Lett. 26, 38-42 (2013)

9. Liu, B., Tunc, C.: Pseudo almost periodic solutions for a class of first order differential iterative equations. Appl. Math. Lett. 40, 29-34 (2015)

10. Shao, J.: Pseudo almost periodic solutions for a Lasota-Wazewska model with an oscillating death rate. Appl. Math. Lett. 43, 90-95 (2015)

11. Long, F.: Positive almost periodic solution for a class of Nicholson's blowflies model with a linear harvesting term. Nonlinear Anal., Real World Appl. 13, 686-693 (2012)

12. Liu, B.: New results on the positive almost periodic solutions for a model of hematopoiesis. Nonlinear Anal., Real World Appl. 17, 252-264 (2014)

13. Fink, A.M.: Almost Periodic Differential Equations. Lecture Notes in Mathematics, vol. 377. Springer, Berlin (1974)

14. Zhang, C.: Almost Periodic Type Functions and Ergodicity. Science Press, Beijing; Kluwer Academic, Dordrecht (2003)

15. Chen, X., Lin, F.: Almost periodic solutions of neutral functional differential equations. Nonlinear Anal., Real World Appl. 11, 1182-1189 (2010) 